\title{
Article
}

\section{Optical Oxygen Sensing with Artificial Intelligence}

\author{
Umberto Michelucci ${ }^{1}$, Michael Baumgartner ${ }^{2}$ and Francesca Venturini ${ }^{1,2, *}$ \\ 1 TOELT LLC, Birchlenstr. 25, 8600 Dübendorf, Switzerland; umberto.michelucci@toelt.ai \\ 2 Institute of Applied Mathematics and Physics, Zurich University of Applied Sciences, Technikumstrasse 9, \\ 8401 Winterthur, Switzerland; bamm@zhaw.ch \\ * Correspondence: vent@zhaw.ch
}

Received: 3 January 2019; Accepted: 10 February 2019; Published: 14 February 2019

check for updates

\begin{abstract}
Luminescence-based sensors for measuring oxygen concentration are widely used in both industry and research due to the practical advantages and sensitivity of this type of sensing. The measuring principle is the luminescence quenching by oxygen molecules, which results in a change of the luminescence decay time and intensity. In the classical approach, this change is related to an oxygen concentration using the Stern-Volmer equation. This equation, which in most cases is non-linear, is parameterized through device-specific constants. Therefore, to determine these parameters, every sensor needs to be precisely calibrated at one or more known concentrations. This study explored an entirely new artificial intelligence approach and demonstrated the feasibility of oxygen sensing through machine learning. The specifically developed neural network learns very efficiently to relate the input quantities to the oxygen concentration. The results show a mean deviation of the predicted from the measured concentration of $0.5 \%$ air, comparable to many commercial and low-cost sensors. Since the network was trained using synthetically generated data, the accuracy of the model predictions is limited by the ability of the generated data to describe the measured data, opening up future possibilities for significant improvement by using a large number of experimental measurements for training. The approach described in this work demonstrates the applicability of artificial intelligence to sensing technology and paves the road for the next generation of sensors.
\end{abstract}

Keywords: artificial intelligence; neural network; machine learning; oxygen sensor; luminescence; optical sensor; luminescence quenching; phase fluorimetry

\section{Introduction}

The determination of oxygen partial pressure is of great interest in numerous areas including medicine, biotechnology, and chemistry. Since oxygen, or better dioxygen, plays an important role in many processes, applications include biomedical imaging, packaging, environmental monitoring, process control, and chemical industry, to mention only a few.

Different methods are used to determine oxygen concentration depending on the application. Among these, optical methods are particularly attractive because they do not consume oxygen, and are therefore reversible, have a fast response time, and allow a good precision and accuracy. Additionally, optical sensors can be manufactured with small sizes, mounted on a fiber and allow therefore both remote and in-situ measurements. An extensive review of optical methods for oxygen sensing and imaging can be found in [1].

A well-known approach, successfully industrialized for several years, is based on the quenching of luminescence by the oxygen molecules [2]. A dye molecule, also called here indicator, is embedded in a matrix permeable for oxygen. Its luminescence is quenched due to the dynamical collisions with oxygen. This process leads to a reduction by an amount which depends on the oxygen concentration 
of both the intensity and decay time of the luminescence [3-5]. An overview of commercially available oxygen sensors based on luminescence quenching can be found in [6].

Sensors based on this principle rely on approximate empirical models to parameterize the dependence of the sensing quantity (e.g., intensity or decay time) on influencing factors. In addition to oxygen concentration, for example, temperature strongly influences the measurement, since both the luminescence and the quenching phenomena are temperature dependent. Other factors impacting the sensing quantity may be sensor specific and affected by the fabrication characteristics. These include, for example, the quenching rate constant of the indicator and the solubility of oxygen in the matrix which serves as a solvent for it. As a result, the characteristic is specific to the system used [7-12].

As described in detail in the next section, the conventional approach consists in using a complex and frequently empirical multi-parametric model to calculate the oxygen concentration from the sensing quantities. Additionally, hardware-related factors, due to for example mechanical and optoelectronic tolerances, must be considered when implementing a luminescence-quenching scheme for commercial applications. The complexity of the model depends on the accuracy and working range that is sought. To reach the accuracy of commercial sensors [6], not only the dependence of the oxygen concentration from the measured quantity has to be approximated analytically, but also the non-linear dependence of these parameters from their influencing factors, e.g., temperature, has to be included. This impacts on the requirements for the electronics performing the calculation. Frequently, a compromise between accuracy, choice of the implementation microelectronic platform, costs and rapidity of the measurements has to be made.

In this study, the application of a machine learning algorithm to luminescence quenching was explored for the first time. To the best of the author's knowledge, machine learning has been applied to time-resolved luminescence data [13], but never to phase-fluorimetry based luminescence sensing. The proposed novel approach consists in using a neural network, which learns to relate the sensed quantities to an oxygen concentration value. This study explored but not exhausted the possibilities of this approach. For example, the training of the neural network was performed with an artificially-created training dataset due to the limited number of measurements available. Additionally, temperature, which needs to be considered in luminescence measurements, was kept constant but could be a parameter predicted by the neural network.

The best-performing network was identified through the study of different architectures and hyperparameter tuning. The trained network was then applied to experimental data, to check its generalization capacity when applied to unseen data. The analysis of the error in the prediction of the oxygen concentrations showed that it was due to the synthetic training data, which were calculated with an approximate analytical model. Using for the training dataset experimental data would therefore reduce the absolute error that can be achieved with this approach. The present study showed that, while the concept as implemented already achieves results comparable with many high-concentration (up to air concentration) commercial sensors and compact sensors based on the classical approaches $[6,14]$, there is still the potential to improve its accuracy. The advantage of the proposed approach is that the analytical model, and therefore its complexity, do not play a role. As long as enough data are available to train the neural network, it will be able to the learn dependence of the oxygen concentration from all parameters.

The paper is organized as follows. Section 2 describes analytical models for oxygen luminescence quenching. Section 3 illustrates the experimental setup used for the experiments. The neural network and its tuning are discussed in Section 4. The results are discussed in Section 5.

\section{Theoretical Model for the Luminescence Quenching}

Oxygen-quenching luminescence sensors are based on the decrease of the luminescence intensity and decay time of the indicator as a function of $\mathrm{O}_{2}$ concentration. In the presence of molecular oxygen, the luminescence of the indicator is quenched because of the radiationless deactivation process due to the interaction of the indicator with molecular oxygen (collisional quenching). In the case of 
homogeneous media characterized by an intensity decay which is a single exponential, the decrease in intensity and lifetime are both described by the Stern-Volmer (SV) equation [3,4]

$$
\frac{I_{0}}{I}=\frac{\tau_{0}}{\tau}=1+K_{S V} \cdot\left[O_{2}\right]
$$

where $I_{0}$ and $I$ are the luminescence intensities in the absence and presence of oxygen, respectively; $\tau_{0}$ and $\tau$ are the decay times in the absence and presence of oxygen, respectively; $K_{S V}$ is the Stern-Volmer constant; and $\left[\mathrm{O}_{2}\right]$ indicates the oxygen concentration.

In many practical applications, the indicator is embedded in a matrix or substrate, frequently a polymer. In this case, the SV curve $I_{0} / I\left(\left[\mathrm{O}_{2}\right]\right)$ deviates from the linear behavior of Equation (1) [1]. The deviation is attributed, for example, to heterogeneities of the microenvironment of the luminescent indicator, or the presence of static quenching. To explain this behavior, several models have been proposed. The simplest scenario involves the presence of at least two environments, in which the indicator is quenched at different rates, often referred to as the multi-site, or for two sites the two-site, model [15]. In this model, the SV curve is the sum of at least two contributions and written as

$$
\frac{I_{0}}{I}=\left(\frac{f_{1}}{1+K_{S V 1} \cdot\left[O_{2}\right]}+\frac{f_{2}}{1+K_{S V 2} \cdot\left[O_{2}\right]}\right)^{-1}
$$

where $I_{0}$ and $I$, respectively, are the luminescence intensities in the absence and presence of oxygen, $f_{1}$ and $f_{2}=1-f_{1}$ are the fractions of the total emission for each component under unquenched conditions, $K_{S V 1}$ and $K_{S V 2}$ are the associated Stern-Volmer constants for each component, and $\left[\mathrm{O}_{2}\right]$ indicates the oxygen concentration. Since $f_{1}+f_{2}=1$, the following notation is used in this work: $f_{1}=f$ and $f_{2}=1-f$. A simplification of this model is that one of the sites is not quenched and therefore the constant $K_{S V 2}$ is zero [9]. Although this model was introduced for luminescence intensities, it is frequently also used to describe the oxygen dependence of the decay times $[3,16]$. Several other more complex models have been proposed [16-19] but are not discussed here.

The luminescence decay time determination is frequently preferable to intensity measurement in sensor implementation because of the proved higher reliability and robustness, since it is not affected by changes in the source intensity or detector sensitivity $[4,20]$. Two approaches can be used to realize decay-time measurement: using a pulsed excitation (time domain) or modulating the intensity of the excitation (frequency domain). The latter, also known as phase fluorimetry, has the advantage of allowing very simple and low-cost realization and is widely used in commercial applications. Therefore, it was chosen for this study. In this approach, the intensity of the excitation light is modulated. The emitted luminescence light is also modulated but shows a phase shift $\theta$ due to the finite lifetime of the excited state. For a single-exponential decay, the relation between these quantities is

$$
\tan \theta=\omega \tau
$$

where $\omega$ is the angular frequency. The range of modulation frequencies to be chosen to determine the intensity decay depends on the lifetimes. The useful modulation frequencies must be high enough so that the phase shift is frequency dependent, but lower than the frequencies where the modulation is no longer measurable.

In the multi-site model, the intensity decay curve is typically no longer a single-exponential decay, even if the decay constant for all sites is the same due to, for example, site-dependent variations of the oxygen diffusion rate [21]. A sum of two or more exponentials can well describe the experimental response time of the system to a pulsed excitation $[8,16]$. In the case of a multi-exponential behavior, there is not one single decay time and the relationship between phase shift and decay times must be calculated through the sine and cosine transforms of the intensity decay and the analytical model becomes significantly more complicated $[4,20,22-24]$. Inherent problems of this type of models are: (1) they may lack relevant physical interpretation when it comes to describing the effect of quenching [8]; 
and (2) calculating the oxygen concentration using a non-linear fit procedure to determine all the parameters may become too complex for a robust solution, as required in a sensor.

In most industrial and commercial sensor applications, where the purpose is to calculate the oxygen concentration, it is standard practice to relate the phase shift measured at a single frequency to an apparent or average lifetime using Equation (3). Combining Equation (3) and assuming the SV relation of Equation (2) to hold for the decay times, the phase shift and the oxygen concentration results described by the approximate model

$$
\frac{\tan \theta_{0}}{\tan \theta}=\left(\frac{f}{1+K_{S V 1} \cdot\left[O_{2}\right]}+\frac{1-f}{1+K_{S V 2} \cdot\left[O_{2}\right]}\right)^{-1}
$$

where $\theta_{0}$ and $\theta$, respectively, are the phase shifts in the absence and presence of oxygen, $f$ and $1-f$ are the fractions of the total emission for each component under unquenched conditions, $K_{S V 1}$ and $K_{S V 2}$ are the associated Stern-Volmer constants for each component, and $\left[\mathrm{O}_{2}\right]$ indicates the oxygen concentration. The quantities $f, K_{S V 1}$, and $K_{S V 2}$ may result frequency dependent, an artifact of the approximation of the model. Since the purpose of this work is to generate synthetic data to perform the training of the neural network, the model of Equation (4) is chosen to describe the data, being as simple as possible, and keeping in mind the limited physical meaning.

Another effect that should be included in the model is the temperature dependence of both the unquenched and the quenched lifetimes. As a result, the parameters $\theta_{0}, K_{S V 1}$, and $K_{S V 2}$ should be characterized by different temperature dependencies.

\section{Experimental Setup}

To determine the parameters for the synthetic data for the training of the neural network and for the validation of the method, several luminescence measurements were performed under varying conditions.

The sample used for the characterization and test is a commercially available Pt-TFPP-based oxygen sensor spot (PSt3, PreSens Precision Sensing GmbH, Regensburg, Germany). To control the temperature of the samples, these were placed in good thermal contact with a copper plate, in a thermally insulated chamber. The temperature of this plate was adjusted at a fixed value between $0{ }^{\circ} \mathrm{C}$ and $45^{\circ} \mathrm{C}$ using a Peltier element and stabilized with a temperature controller (PTC10, Stanford Research Systems, Sunnyvale, CA, USA). The thermally insulated chamber was connected to a self-made gas-mixing apparatus, which enabled varying the oxygen concentration between $0 \%$ and $20 \%$ vol $\mathrm{O}_{2}$ by mixing nitrogen and dry air. In the following, the concentration of oxygen are given in $\%$ of the oxygen concentration of dry air and indicated with \% air. This means, for example, that $20 \%$ air corresponds to $4 \%$ vol $\mathrm{O}_{2}$ and $100 \%$ air corresponds to $20 \%$ vol $\mathrm{O}_{2}$. The absolute error on the oxygen concentration adjusted with the gas mixing device was estimated to be below $1 \%$ air.

The optical setup used in this work for the luminescence measurements is shown schematically in Figure 1.

The excitation light was provided by a $405 \mathrm{~nm}$ LED (VAOL-5EUV0T4, VCC Visual Communications Company, LLC, San Marcos, CA, USA), filtered by a an OD5 short pass filter with cut-off at $498 \mathrm{~nm}$ (Semrock 498 SP Bright Line HC short pass, Semrock, Inc., Rochester, NY, USA) and focused on the surface of the samples with a collimation lens (EO43987, Edmund Optics, Tucson, AZ, USA). The luminescence was focussed by a lens (G063020000, LINOS, Qioptiq, Göttingen, Germany) and collected by a photodiode (SFH 213 Osram, Opto Semiconductors GmbH, Regensburg, Germany). To suppress stray light and light reflected by the sample surface, the emission channel was equipped with an OD5 long pass filter with cut-off at $594 \mathrm{~nm}$ (Semrock 594 LP Edge Basic long pass, Semrock, Inc., Rochester, NY, USA) and an OD5 short pass filter with cut-off at $682 \mathrm{~nm}$ (Semrock 682 SP Bright Line HC short pass, Semrock, Inc., Rochester, NY, USA). The driver for the LED and the trans-impedance amplifier (TIA) are self-made. For the frequency generation and the phase detection 
a two-phase lock-in amplifier (SR830, Stanford Research Inc.) was used. The modulation frequency was varied between $200 \mathrm{~Hz}$ and $20 \mathrm{kHz}$.

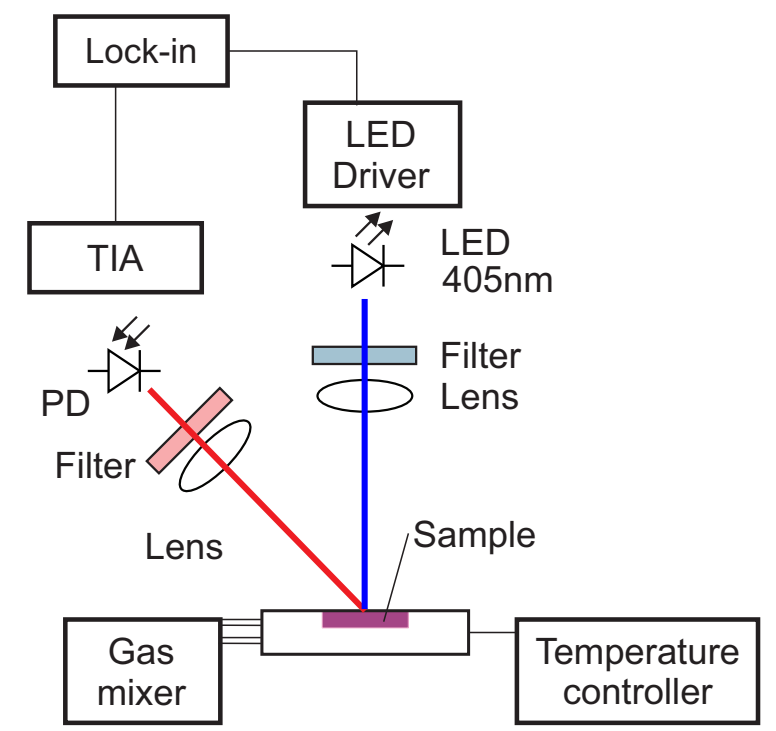

Figure 1. Scheme of the optical experimental setup. Blue is the excitation, red the luminescence optical path. PD, photodiode; TIA, trans-impedance amplifier.

\section{Neural Network Approach}

As described in Section 2, the intensity decay dependence from the relevant quantities, oxygen concentration, temperature and modulation frequency, is quite complex. The approximated mathematical models described in the literature invariably fail to cover all the details of the measurement setup or sensor. To overcome the limitations of the classical methods, which are based on theoretical models, this work proposes a new machine learning approach where the mathematical description and the physical significance of the model describing the luminescence decay are irrelevant. With the help of an optimized feed-forward neural network, the sensor learns to relate an input measured quantity to an output quantity from a large number of examples. In other words, the sensor can be considered as a black-box that transforms the input, the phase shift of Equation (4) measured at several frequencies and a given fixed temperature, into an output, namely the oxygen concentration.

To better describe the method let us introduce the quantity

$$
r\left(\omega, T,\left[O_{2}\right]\right) \equiv \frac{\tan \theta\left(\omega, T,\left[O_{2}\right]\right)}{\tan \theta\left(\omega, T,\left[O_{2}\right]=0\right)}
$$

where the meaning of the symbol is the same as described before. The method consists in taking a certain (possibly large) number $m$ of measurements of the ratio in Equation (5) (this dataset is indicated with $S$ ) at various known values of the frequency and for a set of values of the oxygen concentration sampled from a uniform distribution in the range of interest and use it to train a neural network. The proposed approach is radically different from the commonly used calibration procedures. Usually, the oxygen concentration dependence on the phase shift is programmed in the sensor firmware or in an electronic device as a parametric analytical model. The device-specific parameters are then determined and stored through a calibration.

To demonstrate the feasibility of the approach, the authors used synthetic data for training since a sufficiently large number of experimental data could not be acquired at the time of this work. The next step will be the development of a laboratory setup with the ability to acquire a sufficiently large number of data under varying conditions, e.g., oxygen concentration, modulation frequency, and temperature. A further generalization, which would be possible with a larger set of data, is to extend the neural network to give as output both oxygen concentration and temperature. 
In the next subsections, the overview of the method, the generation of the training data and the details of the neural network model are described.

\subsection{Overview of the Method}

The schematic overview of the method used is shown in the flowchart of Figure 2. The approach can be divided into the following steps:

(i) Acquire data for different values of frequency, temperature, and oxygen concentration.

(ii) Determine a numerical approximation, via interpolation, of the quantities $K_{S V 1}(\omega), K_{S V 2}(\omega)$, and $f(\omega)$ at the chosen temperature $T_{1}$.

(iii) Create the dataset $S$ with $m$ synthetic measurements using the numerical approximation.

(iv) Split the dataset $S$ into a training dataset $S_{\text {train }}$ composed of $80 \%$ of the observations, and a development $S_{d e v}$ dataset composed of $20 \%$ of the observations.

(v) Train several neural network models on the artificial training dataset $S_{\text {train }}$.

(vi) Check for a high-variance (or overfitting) using $S_{t r a i n}$ and $S_{\text {dev }}$ datasets.

(vii) Apply the trained neural network model to the experimental dataset to predict the oxygen concentration and comparison with the measured $\left[\mathrm{O}_{2}\right]$ quantities.

The steps of the data generation are not essential to the method, but rather are necessary if the availability of the experimental data is limited.

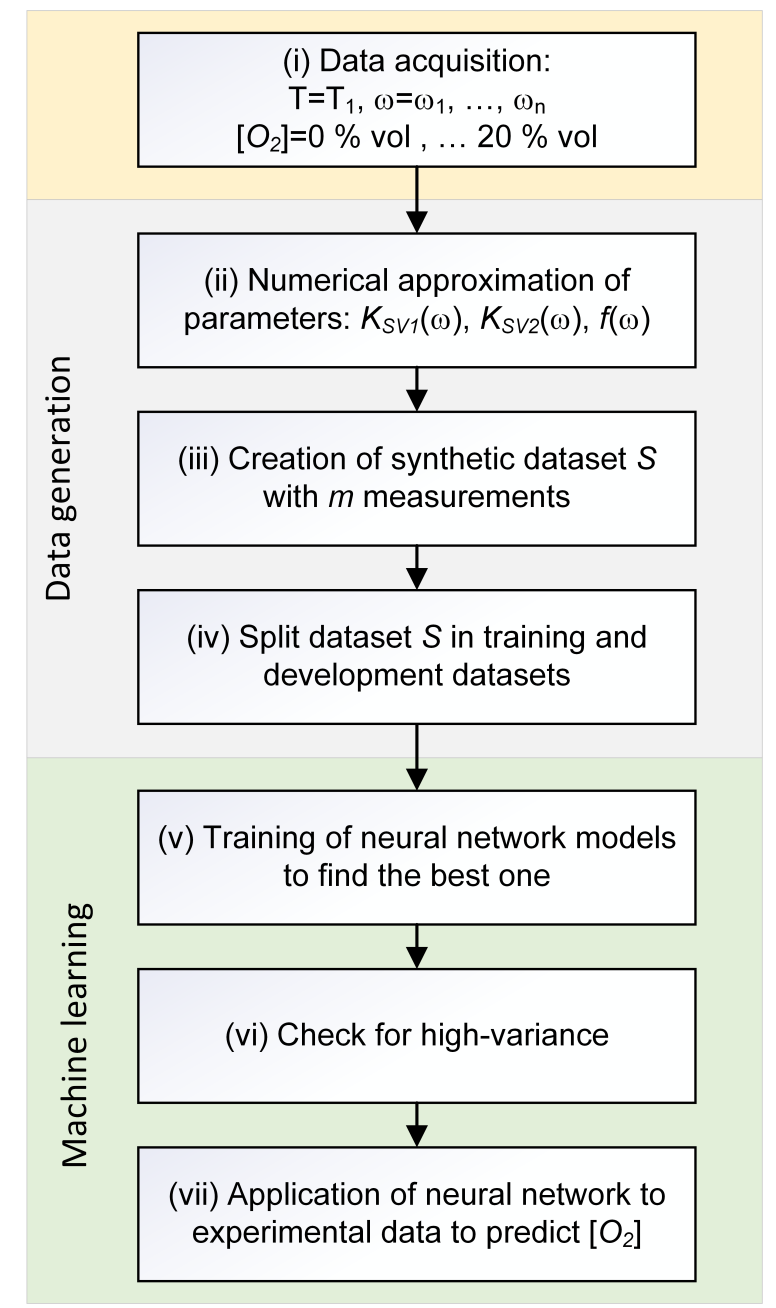

Figure 2. Schematic overview of the steps of the machine learning approach. 


\subsection{Analysis of the Raw Data and Numerical Approximation}

The first step of the method (see Figure 2) consists in the acquisition of the data at a given temperature for various modulation frequencies and oxygen concentrations. In this study, the ratio defined in Equation (5) was measured at sixteen modulation frequencies, between $500 \mathrm{~Hz}$ and $16 \mathrm{kHz}$, ten values of the oxygen concentration, between $0 \%$ air and $100 \%$ air, and five temperatures, between $5{ }^{\circ} \mathrm{C}$ and $45^{\circ} \mathrm{C}$. The measurements at frequencies lower than $500 \mathrm{~Hz}$ were not considered because of the very small value the phase shift of Pt-TFPP assumes. Above $16 \mathrm{kHz}$ the intensity of the modulated light is significantly reduced, which results in higher noise in the phase. Therefore, those frequencies were also not used in this study.

As an example, the phase shifts measured at a fixed modulation frequency of $6 \mathrm{kHz}$ as a function of the oxygen concentration are plotted as $\tan \theta_{0} / \tan \theta$ in Figure 3 for two temperatures.

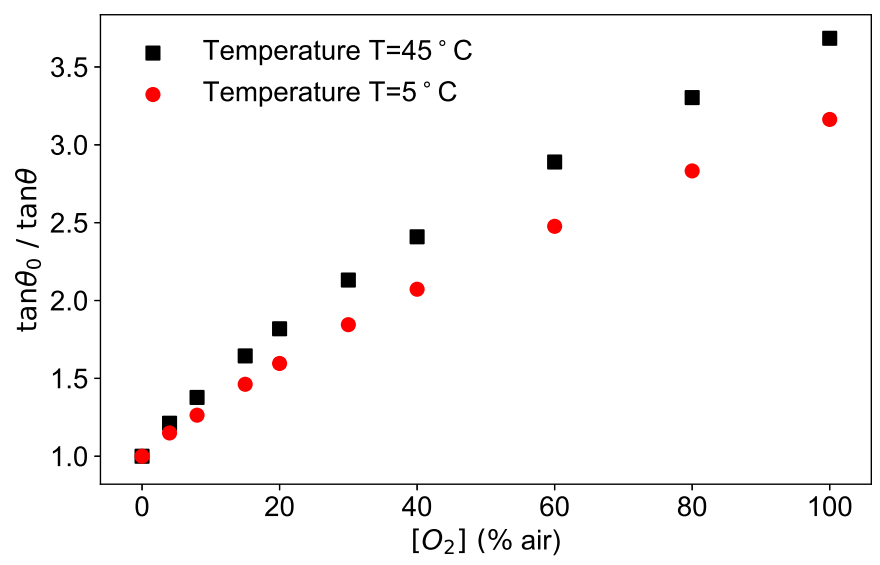

Figure 3. Phase shift measured for modulation frequency $2 \pi \omega=6 \mathrm{kHz}$, at two different temperatures $\left(5^{\circ} \mathrm{C}\right.$ and $\left.45^{\circ} \mathrm{C}\right)$ as a function of the oxygen concentration.

The figure shows the typical dependence of the phase shift on two of the parameters at disposal, oxygen concentration and temperature.

The frequency dependence of the phase shift is shown in Figure 4 for three different concentrations for a fixed temperature of $45{ }^{\circ} \mathrm{C}$. As can be seen in the figure, the ratio $\tan \theta_{0} / \tan \theta$ shows a frequency dependence, which is stronger the higher the concentration is. This dependence is due to the oversimplification of Equation (3), which is approximately correct in the absence of oxygen, and therefore without quenching, but does not hold for higher concentrations.

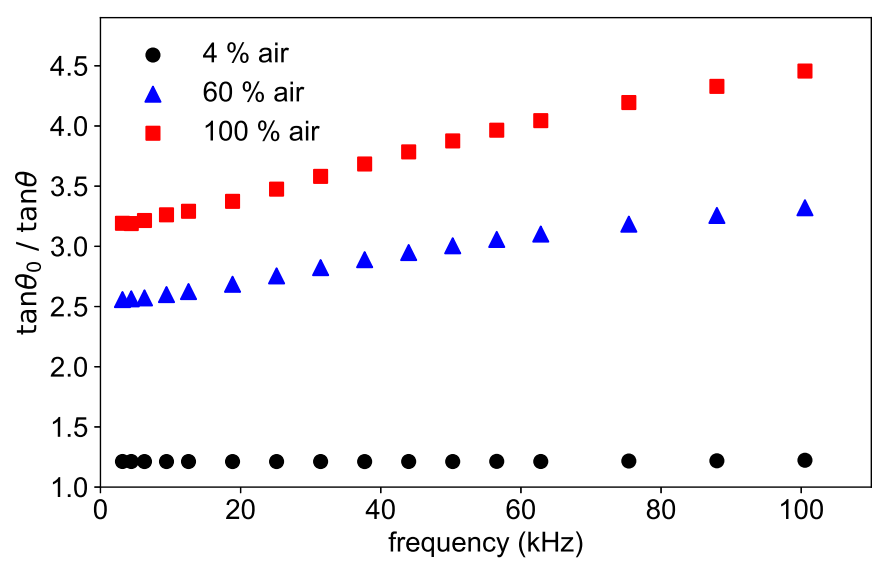

Figure 4. Phase shift measured at three different oxygen concentrations at the temperature $T=45^{\circ} \mathrm{C}$ as a function of the modulation frequency. 
The second step of the method (see Figure 2) consists in the determination of the best numerical approximation of the parameters of the theoretical model. This step is required to generate a large number of data for the training of the neural network. Ideally, as mentioned above, this step should be replaced by a large number of experimental data, which were not available for this work. It is also to be noticed that the physical meaning of equations and parameters used to generate the synthetic data is not relevant and, therefore, is not discussed here.

For this purpose a numeric approximation for the functions $f(\omega, T), K_{S V 1}(\omega, T)$, and $K_{S V 2}(\omega, T)$ was determined by fitting the experimental data according to the model of Equation (4) via standard non-linear fitting procedures. For simplicity, in this work, the temperature was kept constant during the analysis.

\subsection{Generation of Artificial Training Data}

After the determination of the numerical approximation of the parameters, Equation (4) was used to generate a large number of synthetic data for $r\left(\omega, T,\left[O_{2}\right]\right)$, which are needed for the training of the neural network (Step (iii) in Figure 2). To facilitate the implementation in the programming language Python $^{\mathrm{TM}}$, it is advantageous for the parameters $f(\omega, T), K_{S V 1}(\omega, T)$, and $K_{S V 2}(\omega, T)$ to be functions defined on a continuous domain rather than on a discrete number (sixteen) of modulation frequencies. For this purpose, a spline of the third order using the function interp1d from the package scipy [25] of the programming language Python was implemented. The frequency dependence of the parameters $f(\omega, T), K_{S V 1}(\omega, T)$, and $K_{S V 2}(\omega, T)$, as well as the spline used in the code, are shown in Figure 5.
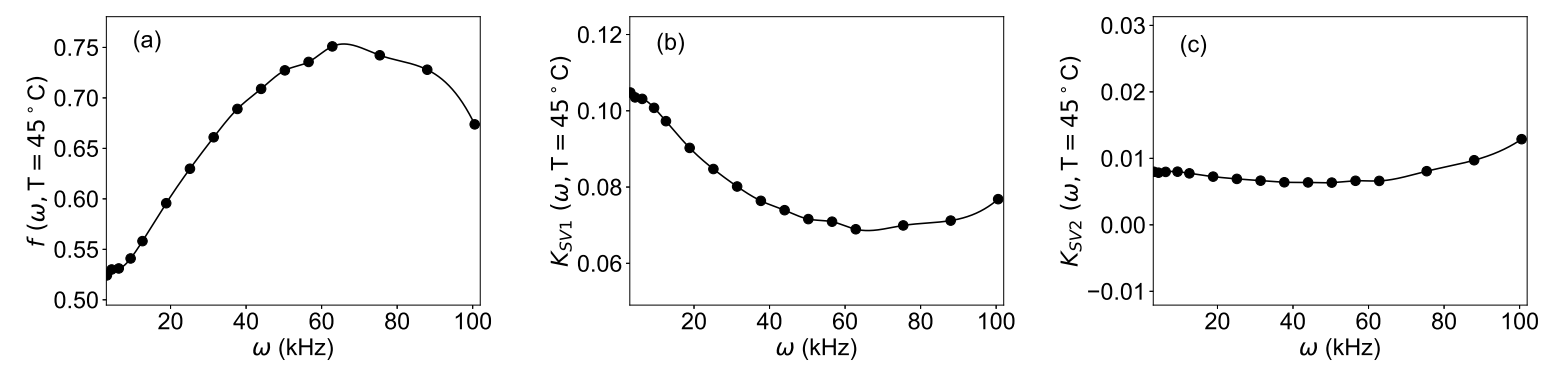

Figure 5. Dependence of the parameters of Equation (4) from the angular frequency of the modulation: (a) $f(\omega, T)$, (b) $K_{S V 1}$, (c) $K_{S V 2}$. Dots: result of the fit of the experimental data; solid line: spline approximation used to generate the synthetic data.

The goal of the network is to predict the oxygen concentration from an array of values of $r\left(\omega, T,\left[O_{2}\right]\right)$ evaluated at a discrete set of sixteen $\omega_{i}$, with $i=1, \ldots, 16$, that have been used for the measurements. Each array $\mathbf{r}=\left(r_{1}, r_{2}, \ldots, r_{16}\right)$ with $r_{i}=r\left(\omega_{i}, T,\left[O_{2}\right]_{j}\right)$ and $i=1, \ldots, 16$ is called an observation in this paper. Each observation is indicated by a superscript $[j]$. Thus, $r_{i}^{[j]}$ indicates $r=r\left(\omega_{i}, T,\left[O_{2}\right]_{j}\right)$ and $\mathbf{r}^{[j]}=\left(r_{1}^{[j]}, r_{2}^{[j]}, \ldots, r_{16}^{[j]}\right)=\left(r\left(\omega_{1}, T,\left[O_{2}\right]_{j}\right), \ldots, r\left(\omega_{16}, T,\left[O_{2}\right]_{j}\right)\right)$. An observation corresponds to a specific value of the oxygen concentration.

The synthetic data consist of a set $S$ of $m=5000$ observations using oxygen concentration values uniformly distributed between $0 \%$ air and $110 \%$ air. The value of $110 \%$ air was chosen since the neural network predictions tend to be less good when dealing with observations that are close to a boundary. The tests show that, having only observations for the training with $\left[\mathrm{O}_{2}\right]<100 \%$ air makes the network predictions less accurate in the prediction for $\left[\mathrm{O}_{2}\right]$ close to $100 \%$ air. A discussion of this effect can be found in [26].

Next (Step (iv) in Figure 2), these data are split randomly into a training dataset $S_{\text {train }}$ containing $80 \%$ of the data, i.e., 4000 observations, used to train the network, and a development dataset $S_{\text {dev }}$ containing $20 \%$ of the data, i.e., 1000 observations, used to test the generalization of the network when applied to unseen data. For the validation, the neural network model is applied to a test dataset, indicated with $S_{\text {test }}$, whose observations are the ten experimental measurements. Finally, the predictions of the oxygen concentration are compared to the measured values. 


\subsection{Neural Network Model}

The true machine learning (Steps (v-vii) in Figure 2) starts with the neural network model. The building block of the network used in this work is a neuron, which transforms a set of real numbers given as inputs $x_{i}$ in an output $\hat{y}$ using the formula

$$
\hat{y}=\sigma\left(\sum_{\text {number of inputs }} w_{i} x_{i}+b\right)
$$

where $w_{i}$ are called weights, $b$ is bias, and $\sigma$, which is called the activation function, is the sigmoid function that has the analytical form

$$
\sigma(z)=\frac{1}{1+e^{-z}}
$$

This is schematically depicted in Figure 6.

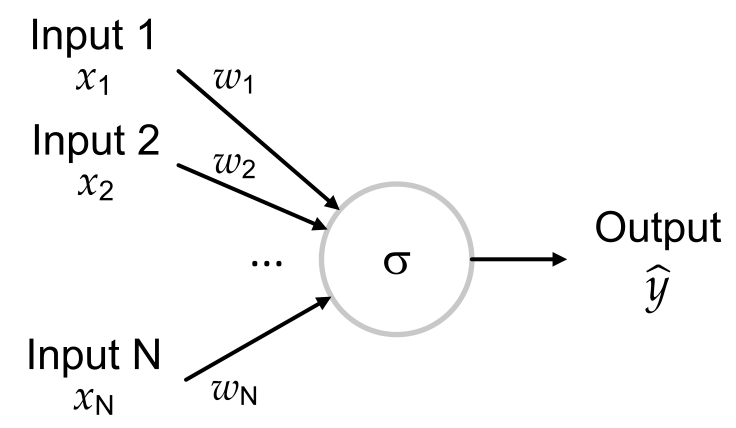

Figure 6. A schematically depicted neuron. It applies a non-linear transformation to the inputs with the sigmoid activation function to obtain its output.

The architecture of the neural network of this work is shown schematically in Figure 7.

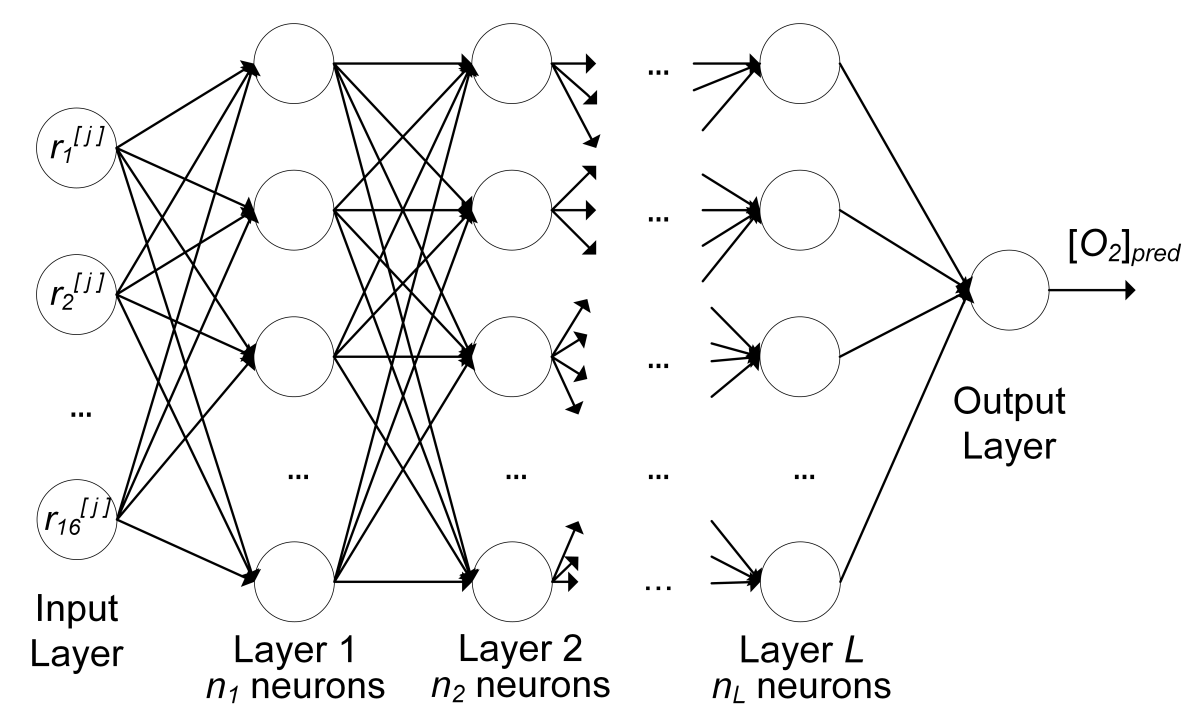

Figure 7. Architecture of the feed-forward neural network with $L$ layers, each having a number of neurons $n_{i} . \quad r_{i}^{[j]}$ is the $i$ th feature of the $[j]$ th observation; the output is the predicted oxygen concentration $\left[\mathrm{O}_{2}\right]_{\text {pred }}$.

The network includes a number of layers $L$, each with the same number of neurons $n_{i}$. The architecture in Figure 7 is of the type feed-forward, where each neuron in each layer gets as 
input the output of all neurons in the previous layer before, and feeds its output to each neuron in the subsequent layer.

A neural network model is made of three parts: the network architecture, described above (Figure 7), the cost function $J$, and an optimizer. Training the network means finding the best weights and bias of all neurons of the network (cf. Equation (6) for a single neuron) to minimize $J$. The optimizer is the algorithm used to minimize the cost function. Since it is a regression problem, the cost function $J$ is taken to be the mean squared error, defined as the squared average of the absolute value of the difference between the predicted oxygen concentration values and the expected ones. To minimize the cost function, the optimizer Adaptive Moment Estimation (Adam) [27] was used. The implementation was performed using the TensorFlow ${ }^{\mathrm{TM}}$ library.

To study the dependence of the results from the architecture of the network, a process called hyperparameter optimization, both the number of layers $L$ and the number of neurons per layer $n_{i}$ were varied. For all the neurons the sigmoid function was taken as activation function. For the output neuron, that has as output the predicted values $\left[\mathrm{O}_{2}\right]_{\text {pred }}$, the function $110 \cdot \sigma$ was chosen, since the dataset contains training observations with $\left[\mathrm{O}_{2}\right]$ up to $110 \%$ air. The predictions of the network are then compared to identify the best architecture.

The metric used to compare results from different network models is the mean absolute error (MAE), defined as the average of the absolute value of the difference between the predicted and the expected or measured oxygen concentration. For example, for the $S_{\text {test }}$ dataset

$$
\operatorname{MAE}\left(S_{\text {test }}\right)=\frac{1}{\left|S_{\text {test }}\right|} \sum_{S_{\text {test }}}\left|\left[O_{2}\right]_{\text {pred }}-\left[O_{2}\right]_{\text {meas }}\right|
$$

with $\left|S_{\text {test }}\right|$ the size (or cardinality) of the dataset $S_{\text {test }}$. The further quantity used to analyze the performance of the network is the absolute error (AE) of a given observation $j$, defined as the absolute value of the difference between the predicted and the measured $\left[\mathrm{O}_{2}\right]^{[j]}$ value

$$
\mathrm{AE}^{[j]}=\left|\left[\mathrm{O}_{2}\right]_{\text {pred }}^{[j]}-\left[\mathrm{O}_{2}\right]_{\text {meas }}^{[j]}\right| .
$$

\section{Results and Discussion}

To investigate the performance of the neural network, the architecture was varied by changing the number of layers, between 1 and 3, and the number of neurons per layer, between 3 and 50 . The training was performed for all trials with batch gradient descent for $10^{5}$ epochs and learning rate of 0.001. The latter was chosen because of the fast convergence of the cost function. For each trial, the MEA of Equation (8) was calculated. The result of the analysis is summarized in Figure 8.

As expected, the values of all three MAE are larger for neural networks with lower effective complexity, on the left of the plot, than for ones with higher complexity, on the right of the plot, regardless of the dataset to which the network is applied. This result reflects the fact that, if the network is not complex enough, it does not perform well since it cannot learn the subtleties of the data. In Figure $8 \mathrm{a}, \mathrm{b}$, it can be noted that the $\mathrm{MAE}_{\text {train }}$ and $\mathrm{MAE}_{\text {dev }}$ have similar behavior and assume almost the same values, going to zero for increasing complexity. For the architectures studied in this work, both $\mathrm{MAE}_{\text {train }}$ and $\mathrm{MAE}_{\text {dev }}$ reach a minimum of $0.012 \%$ air for the network with 3 layers and 50 neurons. The reason for the error going almost to zero is that both the training and development datasets contained synthetic data generated by the same function, therefore without any experimental noise. Typically, when training neural network models, it is important to check if we are in a so-called overfitting regime. The essence of overfitting is to have unknowingly extracted some of the residual variation (i.e., the noise or errors) as if that variation represented an underlying model structure [28]. In this work, with increasing complexity, the network will never go into such a regime, since the development dataset is a perfect representation of the training dataset. This leads to almost identical $\mathrm{MAE}_{\text {train }}$ and $\mathrm{MAE}_{\text {dev }}$ error values, regardless of the network architecture effective complexity. 


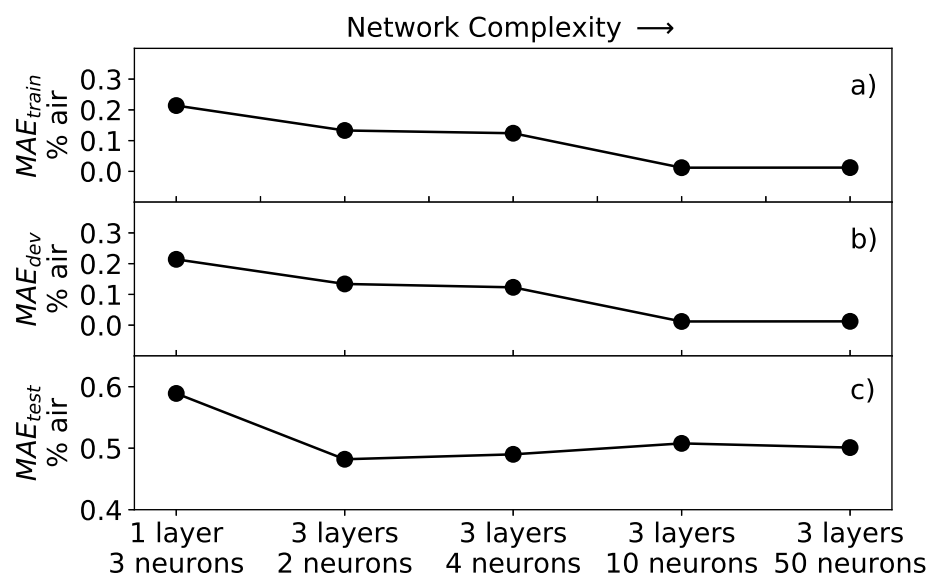

Figure 8. Mean absolute error of the network applied to different datasets for various networks architectures: (a) $\mathrm{MAE}_{\text {train }}$ for training dataset; (b) $\mathrm{MAE}_{\text {dev }}$ for development dataset; and (c) $\mathrm{MAE}_{\text {test }}$ for the test dataset. The complexity of the network increases left to right.

The $\mathrm{MAE}_{\text {test }}$ (Figure 8c), on the other hand, shows a different behavior, rapidly improving from the simplest architecture of one layer and three neurons, but then not further decreasing by increasing the complexity of the neural network. In other words, as soon as the complexity of the network is enough to approximate well the inverse function of Equation (4), the MAE test stabilizes at a value of approximately $0.5 \%$ air.

The reason $\mathrm{MAE}_{\text {test }}$ does not go to zero is twofold. First, $S_{\text {test }}$ includes the experimental measurements, which are affected by an experimental error. Since the neural network was trained with synthetic data, it does not include such an error and will consequently always have a certain deviation in predicting $\left[\mathrm{O}_{2}\right]$.

Second and most importantly, the theoretical model of Equation (4) does not approximate the experimental data sufficiently well, as illustrated in Figure 9. In the top panel, $\tan \theta_{0} / \tan \theta$ measured at a modulation frequency of $6 \mathrm{kHz}$ and the temperature of $45^{\circ} \mathrm{C}$ is shown as a function of the oxygen concentration together with the best fit using Equation (4). The residuals, calculated as the difference between the fit and the measured data, are plotted in the bottom panel of Figure 9 and show that the deviation of the measurement and model increases with higher oxygen concentration. Therefore, the training performed with synthetic data has the disadvantage to lead to a network which learns from a dataset with different functional shape (albeit not extremely so) than the test dataset. This contribution to the $\mathrm{MAE}_{\text {test }}$ could be eliminated using experimental measures as a training dataset, allowing thus to achieve even better predictions of the oxygen concentration than $0.5 \%$ air.

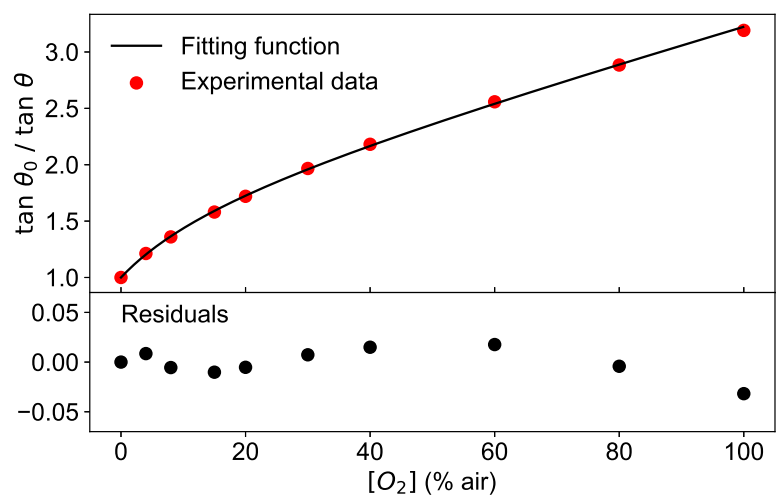

Figure 9. (top) Phase shift measured at a modulation frequency of $6 \mathrm{kHz}$ and at the temperature of $45{ }^{\circ} \mathrm{C}$ for various values of oxygen concentration (circles). The line is the fit obtained with the Equation (4). (bottom) Residuals of the fit. 
These results are supported by the analysis of the dependence of the absolute error AE on the oxygen concentration. As an example, the absolute error calculated as in Equation (9) is shown in Figure 10 for a network with 3 layers and 10 neurons. As shown in Figure 10, the AE is below 0.5\% air for low oxygen concentrations, tends to increase with higher $\left[\mathrm{O}_{2}\right]$ values and reaches its maximal value of $2 \%$ air at $100 \%$ air. This is consistent with the deviations shown in Figure 9, indicating that the fitting function works worst at $100 \%$ air.

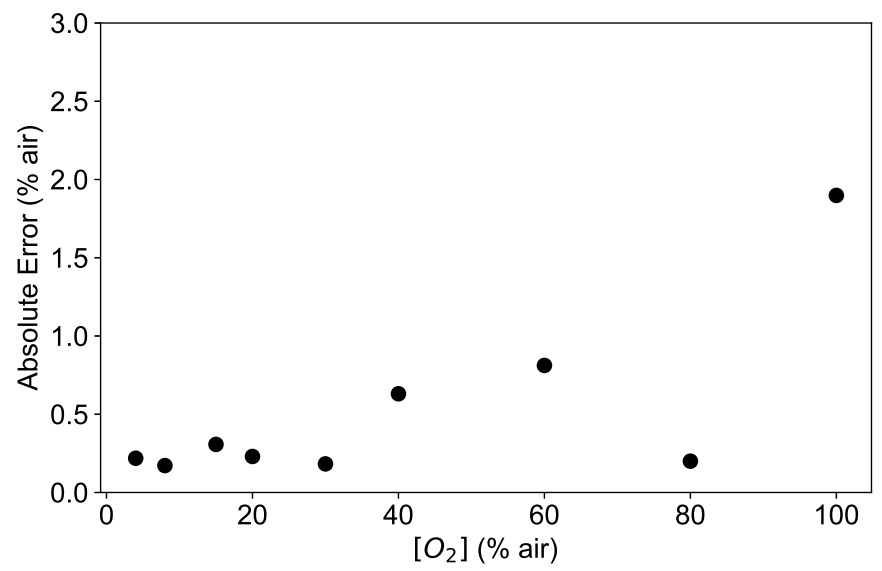

Figure 10. Absolute error (AE) of the neural network model prediction applied to the experimental data at the temperatures $45^{\circ} \mathrm{C}$ for a network with 3 layers and 10 neurons.

The above mentioned observations are valid at all temperatures studied, as shown in Figure 11. For each temperature, the absolute error is calculated for the available oxygen concentrations and displayed as a box plot, where the median is visible as a red line.

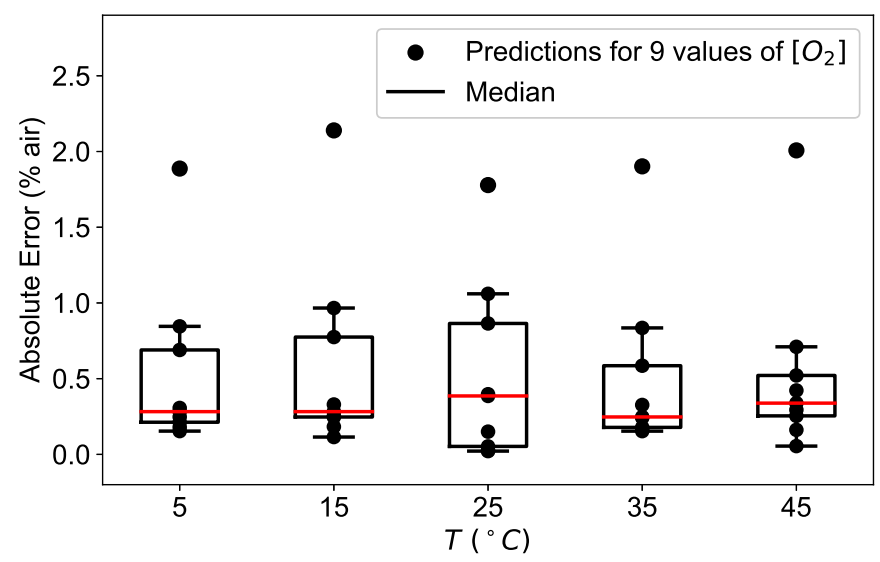

Figure 11. Absolute error (AE) distribution of the neural network model prediction applied to the experimental data for different concentrations calculated at the temperatures of $5{ }^{\circ} \mathrm{C}, 15{ }^{\circ} \mathrm{C}, 25{ }^{\circ} \mathrm{C}$, $35^{\circ} \mathrm{C}$, and $45^{\circ} \mathrm{C}$ for a network with 3 layers and 10 neurons.

For all temperature investigated, the median remains below $0.5 \%$ air for low oxygen concentrations and the absolute error has a maximal value of $2 \%$ air at $100 \%$ air, plotted separately in Figure 11 for clarity.

From a theoretical point of view, a network with one layer can approximate any non-linear function $[29,30]$. However, a network with more layers but fewer neurons in total might be able to capture specific features of the data better and faster, that is with a smaller number of epochs in the training. The analysis of the different network architectures performed in this work supports these results. To reach the same value for the MAE with networks with one layer requires 5-10 times more 
epochs for the training. Consequently, it is much more efficient to choose a network with more layers. In this work, networks with architectures more complex than 3 layers and 50 neurons were also studied. Since the $\mathrm{MAE}_{\text {test }}$ stabilizes already for simple architectures, as shown in Figure 8, an increase in the complexity would not further improve the performance.

\section{Conclusions}

This work explored a new approach to optical luminescence sensing, proving that, to build an accurate oxygen sensor, it is not necessary to implement complex non-linear pseudo-physical models to describe the dependence of the measured quantity, here the phase shift, on the oxygen concentration. The sensor-specific deviations from a simple SV model may be caused, for example, by the method for the immobilization of the indicators in the substrate, or by luminescence from components typically included in a sensor, such as absorption filters or glues. Therefore, to reach a high accuracy, the classical approach requires empirically modeling the dependence of the parameters, $f, K_{S V 1}$, and $K_{S V 2}$, of the inverted SV multi-site model from all the relevant influencing quantities, for example the temperature or the modulation frequency. The resulting algorithms frequently are computationally intensive and per-definition only an approximation. Furthermore, for a commercial solution, it is desirable for the chosen parameterization to be valid for all sensors, with only a few parameters that need to be determined during a device-specific calibration. This imposes higher requirements, and therefore costs, on the device components, and may require further compromising on the accuracy. The proposed artificial intelligence approach has the potential to overcome these limitations.

Several neural network architectures were tested, demonstrating that already networks with rather simple structures can predict the oxygen concentration with a mean absolute error $\mathrm{MAE}_{\text {test }}$ of $0.5 \%$ air. By an analysis of discrepancies as a function of the oxygen concentration, it was possible to identify the main contribution to the error. The results show that the absolute error AE increased with increasing concentrations, going from well below $0.5 \%$ air at $30 \%$ air to a maximum of $2 \%$ air at $100 \%$ air. The main contribution to the $\mathrm{AE}$ was identified in the poor agreement of the conventional model describing the quenching of the luminescence, which was used to generate the training data. By performing the training on experimental data, this error is expected to decrease significantly.

This work paves the road to a new and completely different approach in sensor development. Using artificial intelligence is not necessary to define any models with parameters to capture all influencing factors. Once the sensor hardware is given, i.e., all optical, electronic, mechanical and chemical components are assembled, a neural network can be trained to learn to predict one, or possibly more, quantities of interests, in this work the oxygen concentration. The advantages of this approach will impact positively, for example, on the scale-up in sensor fabrication because the requirements on the hardware can be relaxed. The device-specific characteristics will be accounted for by the trained neural network.

Author Contributions: U.M. analyzed the data, developed the neural network and wrote the paper. M.B. built the laboratory setup and performed the experiments. F.V. conceived the study, analyzed the data and wrote the paper.

Funding: This research received no external funding.

Conflicts of Interest: The authors declare no conflict of interest.

\section{Abbreviations}

The following abbreviations are used in this manuscript:

SV Stern-Volmer

LED Light-emitting diode

TIA trans-impedance amplifier

Adam Adaptive moment estimation

MAE Mean absolute error

AE Absolute error 


\section{References}

1. Wang X.-D.; Wolfbeis O.S. Optical methods for sensing and imaging oxygen: Materials, spectroscopies and applications. Chem. Soc. Rev. 2014, 43, 3666-3761. [CrossRef] [PubMed]

2. Narayanaswamy, R.; Wolfbeis, O.S. Optical Sensors: Industrial Environmental and Diagnostic Applications, 1st ed.; Springer Science \& Business Media: Berlin, Germany, 2004; Volume 1, pp. 28-30.

3. Quaranta, M.; Sergey, M.B.; Klimant, I. Indicators for optical oxygen sensors. Bioanal. Rev. 2012, 4, 115-157. [CrossRef] [PubMed]

4. Lakowicz, J.R. Principles of Fluorescence Spectroscopy, 3rd ed.; Springer: Singapore, 2006.

5. Demas, J.N.; DeGraff, B.A.; Coleman, P.B. Oxygen Sensors Based on Luminescence Quenching. Anal. Chem. 1999, 71, 793A-800A. [CrossRef] [PubMed]

6. Wolfbeis, O.S. Luminescent sensing and imaging of oxygen: Fierce competition to the Clark electrode. BioEssays 2015, 37, 921-928. [CrossRef] [PubMed]

7. $\mathrm{Xu}, \mathrm{W}$;; McDonough, R.C.; Langsdorf, B.; Demas, J.N.; DeGraff, B.A. Oxygen sensors based on luminescence quenching: Interactions of metal complexes with the polymer supports. Anal. Chem. 1994, 66, 4133-4141. [CrossRef]

8. Draxler, S.; Lippitsch, M.E.; Klimant, I.; Kraus, H.; Wolfbeis, O.S. Effects of polymer matrixes on the time-resolved luminescence of a ruthenium complex quenched by oxygen. J. Phys. Chem. 1995, 99, 3162-3167. [CrossRef]

9. Hartmann, P.; Trettnak, W. Effects of polymer matrices on calibration functions of luminescent oxygen sensors based on porphyrin ketone complexes. Anal. Chem. 1996, 68, 2615-2620. [CrossRef] [PubMed]

10. Mills, A. Controlling the sensitivity of optical oxygen sensors. Sens. Actuators B Chem. 1998, 51, 60-68. [CrossRef]

11. Dini, F.; Martinelli, E.; Paolesse, R.; Filippini, D.; D’Amico, A.; Lundström, I.; Di Natale, C. Polymer matrices effects on the sensitivity and the selectivity of optical chemical sensors. Sens. Actuators B Chem. 2011, 154, 220-225. [CrossRef]

12. Badocco, D.; Mondin, A.; Pastore, P.; Voltolina, S.; Gross, S. Dependence of calibration sensitivity of a polysulfone/Ru (II)-Tris (4,7-diphenyl-1,10-phenanthroline)-based oxygen optical sensor on its structural parameters. Anal. Chim. Acta 2008, 627, 239-246. [CrossRef]

13. Đorđević, N.; Beckwith, J.S.; Yarema, M.; Yarema, O.; Rosspeintner, A.; Yazdani, N.; Leuthold, J.; Vauthey, E.; Wood, V. Machine Learning for Analysis of Time-Resolved Luminescence Data. ACS Photonics 2018, 5, 4888-4895. [CrossRef]

14. Chu, C.S.; Lin, K.Z.; Tang, Y.H. A new optical sensor for sensing oxygen based on phase shift detection. Sens. Actuators B Chem. 2016, 223, 606-612. [CrossRef]

15. Carraway, E.R.; Demas, J.N.; DeGraff, B.A.; Bacon, J.R. Photophysics and Photochemistry of Oxygen Sensors Based on Luminescent Transition-Metal Complexes. Anal. Chem. 1991, 63, 337-342. [CrossRef]

16. Demas, J.N.; DeGraff, B.A.; Wenying, X. Modeling of Luminescence Quenching-Based Sensors: Comparison of Multisite and Nonlinear Gas Solubility Models. Anal. Chem. 1995, 67, 1377-1380. [CrossRef]

17. Hartmann, P.; Leiner, M.P.; Lippitsch, M.E. Luminescence Quenching Behavior of an Oxygen Sensor Based on a Ru(1l) Complex Dissolved in Polystyrene. Anal. Chem. 1995, 67, 88-93. [CrossRef]

18. Mills, A. Response characteristics of optical sensors for oxygen: A model based on a distribution in $\tau_{0}$ and $k_{q}$. Analyst 1999, 124, 1309-1314. [CrossRef]

19. Chatni, M.R.; Li, G.; Porterfield, D.M. Frequency-domain fluorescence lifetime optrode system design and instrumentation without a concurrent reference light-emitting diode. Appl. Opt. 2009, 48, 5528-5536. [CrossRef]

20. Lippitsch, M.E.; Draxler, S. Luminescence decay-time-based optical sensors: Principles and problems. Sens. Actuators B Chem. 1993, 11, 97-101. [CrossRef]

21. Ogurtsov V.I.; Papkovsky, D.B; Papkovskaia, N.Y. Approximation of calibration of phase-fluorimetric oxygen sensors on the basis of physical models. Sens. Actuators B Chem. 2001, 81, 17-24. [CrossRef]

22. Digris, A.V.; Novikov, E.G.; Apanasovich, V.V. A fast algorithm for multi-exponential analysis of time-resolved frequency-domain data. Opt. Commun. 2005, 252, 29-38. [CrossRef]

23. Stehning, C.; Holst, G.A. Addressing multiple indicators on a single optical fiber-digital signal processing approach for temperature compensated oxygen sensing. IEEE Sens. J. 2004, 4, 153-159. [CrossRef] 
24. Ogurtsov, V.I.; Papkovsky, D.B. Application of frequency spectroscopy to fluorescence-based oxygen sensors. Sens. Actuators B Chem. 2006, 113, 608-616. [CrossRef]

25. Jones, E.; Oliphant, E.; Peterson, P. SciPy: Open Source Scientific Tools for Python. Available online: http:/ / www.scipy.org/ (accessed on 26 December 2018).

26. Michelucci, U. Applied Deep Learning-A Case-Based Approach to Understanding Deep Neural Networks; Apress Media, LLC: New York, NY, USA, 2018; pp. 374-375. ISBN 978-1-4842-3789-2.

27. Kingma, D.P.; Adam, J.B. A method for stochastic optimization. arXiv 2014, arXiv:1412.6980.

28. Burnham, K.P.; Anderson, D.R. Model Selection and Multimodel Inference, 2nd ed.; Springer: New York, NY, USA, 2002.

29. Montufar, G.F.; Pascanu, R.; Cho, K.; Bengio, Y. On the Number of Linear Regions of Deep Neural Networks. Available online: https:/ / papers.nips.cc/paper/5422-on-the-numberof-linear-regions-of-deepneural-networks.pdf (accessed on 26 December 2018).

30. Fortuner, B. Can Neural Networks Solve Any Problem? Available online: https:/ /towardsdatascience.com/ can-neural-networksreally-learn-any-function-65e106617fc6 (accessed on 27 November 2018).

(c) 2019 by the authors. Licensee MDPI, Basel, Switzerland. This article is an open access article distributed under the terms and conditions of the Creative Commons Attribution (CC BY) license (http://creativecommons.org/licenses/by/4.0/). 